\title{
Entwicklung von Krebs-Screening- Programmen in der Schweiz
}

\section{Philip Went}

PD Dr. med., Vorstandsmitglied und Präsident der Tarifkommission der SGPath; Chefarzt, Institut für Pathologie des Kantonsspitals Graubünden

Krebs-Screening-Programme sollten in der gesamten Schweiz einheitlich standardisiert durchgeführt werden. Die Struktur des Gesundheitswesens in der Schweiz führt allerdings zu kantonal voneinander abweichenden Programmen.

Kolorektale Karzinome sind in der Schweiz die dritthäufigsten Karzinome in Bezug auf Inzidenz und Mortalität bei Männern und Frauen. Durch einfache, im Vergleich zur Therapie billige, und risikoarme Untersuchungen kann diese häufige Erkrankung nicht nur geheilt, sondern noch häufiger komplett vermieden

\section{Es sollte ein einheitlicher Prozess mit schweizweit einheitlichen Entschädigungen etabliert werden.}

werden. Es werden deshalb schweizweit Screening-Programme entwickelt, wobei deutliche Unterschiede zu anderen Screening-Programmen bestehen: Der Nutzen bei kolorektalen Karzinomen ist unbestritten (Differenz zu Mammakarzinom), und der Screening-Prozess ist noch nicht etabliert (Unterschied zu Portiokarzinom-Screening). Wie aber werden diese Programme nun in der Schweiz entwickelt?

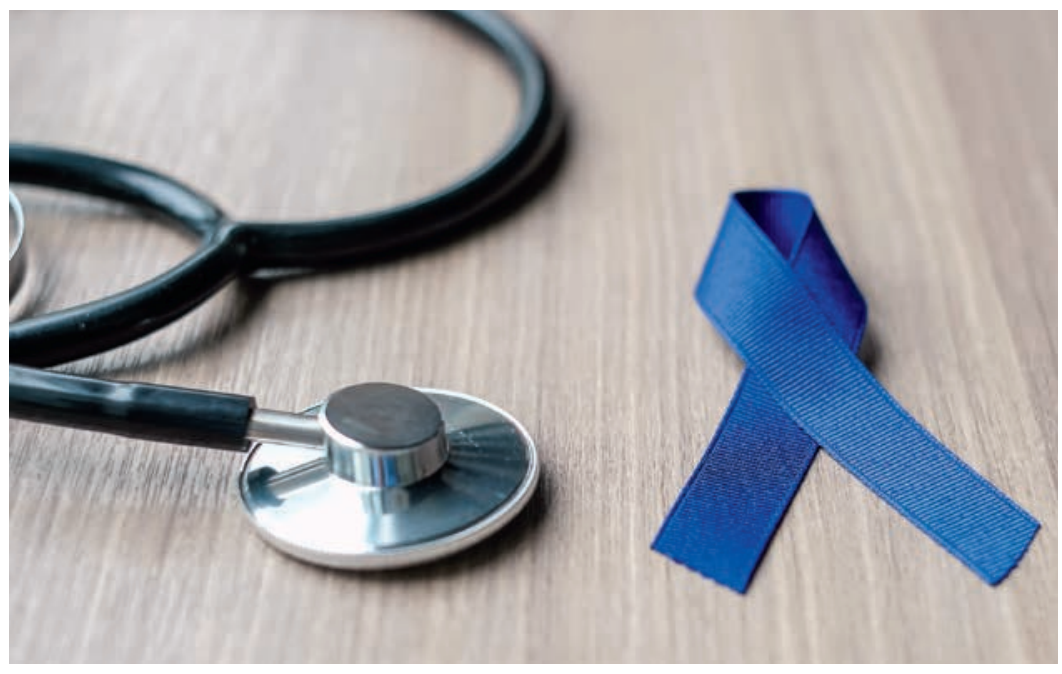

Im Unterschied zu anderen Krebsarten ist der Screening-Prozess bei kolorektalen Karzinomen noch nicht etabliert.
Es sollte ein in Bezug auf Anfang und Ende des Programms einheitlicher Prozess mit schweizweit einheitlichen Entschädigungen etabliert werden. Dies benötigt einheitliche Rahmenbedingungen, eine klare Leitung und, nicht zuletzt, ein klares Ziel. Die Vorgabe erscheint einfach, in erster Linie handelt es sich um, zugegebenermassen nicht einfache, logistische Probleme. Natürlich müssten dazu Gespräche mit den verschiedensten Akteuren geführt werden.

\section{Noch kein einheitlicher Prozess}

Die Erfahrung von Beteiligten zeugt nun aber von einem etwas anderen Geschehen: In erster Linie entwickelt jeder Kanton losgelöst sein eigenes Programm. Dieses wird häufig von der kantonalen Krebsliga im Auftrag des jeweiligen Gesundheitsdepartements, welches den Kostenrahmen vorgibt, organisiert. Gleichzeitig definiert die Dachorganisation Swiss Cancer Screening ein Rahmen-Screening-Programm nach Vernehmlassung und unter Teilnahme der involvierten Gesellschaften, zum Beispiel unter Beteiligung der SGPath, und weiterer Organisationen. Alle diese verschiedenen Aktivitäten sind zeitlich und inhaltlich mässig aufeinander abgestimmt, so dass in der Schweiz zum heutigen Tag verschiedene Programme bestehen, welche sich zum Beispiel in Bezug auf den Endpunkt des Programms unterscheiden. Wichtige Akteure wurden allerdings initial vergessen und mussten sich teilweise selbst einbringen. Auch die Einschlusskriterien der einzelnen Kantone sind nicht absolut deckungsgleich. Dies muss logischerweise dazu führen, dass auch die Kosten schlecht vergleichbar sein werden, was die weitere Entwicklung, z.B. die nationale Standardisierung der Programme, sehr effizient verhindern wird. 


\section{Bislang keine standardisierten Entschädigungen}

Zur Festlegung der Entschädigung ist zudem Folgendes festzuhalten: Die Kosten des Screenings müssen laut KVG von den Versicherern übernommen werden und sind franchisenbefreit. Dies bedeutet, dass Preise ausserhalb des ambulanten Tarifsystems (oder der Analysenliste) verhandelt werden können, was unserer Ansicht nach eine grosse Chance zur Weiterentwicklung und Festlegung von verbesserten, fairen und transparenten Entschädigungen darstellt. Prozedural muss der Programmleiter die Entschädigungen mit den Kostenträgern vereinbaren und dann zur Genehmigung dem Kanton vorlegen. Die Leistungserbringer müssen hier nicht zwingend involviert werden, dies wurde konkret in mehreren Kantonen auch vermieden. Anschliessend werden die Leistungserbringer von den Programmleitern ernannt, wobei diese dann die im Programm definierten Leistungen zu den vorgegebenen Entschädigungen erbringen sollen. Falls vorgängig die Leistungserbringer weder in der Programmfestlegung noch in den Entschädigungsverhandlungen kontaktiert wurden, führt dies naturgemäss zu äusserst unerfreulichen Gesprächen.

\section{Beispiel: Pathologieleistungen}

Anhand von Pathologieleistungen kann auch exemplarisch auf folgendes Problem hingewiesen werden: Pathologen sind häufig an grossen Spitälern angestellt. Letztere haben, wie Figura der letzten Jahre zeigt, sehr grosse Schwierigkeiten, Abrechnungsänderungen zu implementieren: Teilweise konnten von grossen Spitälern während Monaten keine Rechnungen gestellt werden. Falls nun Pathologen im Rahmen des Kolonkarzinom-Screening-Programms involviert

\section{Das Wichtigste in Kürze}

- Kolorektale Karzinome sind in der Schweiz die dritthäufigsten Karzinome in Bezug auf Inzidenz und Mortalität bei Männern und Frauen.

- Das gemeinsame primäre Ziel aller am Screening-Programm Beteiligten ist, die weitgehend verhinderbare hohe Zahl an Kolonkarzinom-Erkrankungen und -Todesfällen in der Schweiz drastisch zu senken.

- Ein zweites, sekundäres Ziel sollte sein, einen zeitgemässen, nationalen Standard in Bezug auf Prozess und Entschädigung zu setzen und damit die Schweiz international zu positionieren.

PD Dr. med. Philip Went philip.went[at]ksgr.ch Tel. +41812566540
- Aufgrund der extrem dezentralisierten Struktur in der Schweiz sollten alle Beteiligten zugunsten dieser grösseren Ziele gemeinsam zusammenarbeiten, Leidtragende sind ansonsten ganz konkret alle Bewohner der Schweiz. sind, muss eine extrem standardisierte Leistung erbracht werden. Es könnte somit auch die Entschädigung standardisiert sein. Nun passiert aber genau das Gegenteil: In jedem Kanton werden andere Entschädigungen ausgehandelt, teilweise versuchen Kostenträger sogar innerhalb eines Kantons verschiedene Preise auszuhandeln. Es gibt nun Institute für Pathologie, welche für mehrere Kantone tätig sind. Das bedeutet konkret, dass diese Institute für die gleiche, sehr standardisierte Leistung vier oder mehr verschiedene Preise verrechnen müssen, welche sich allerdings alle innerhalb einer Bandbreite von wenigen Franken befinden. Die Sekundärkosten, die für diesen Unsinn generiert werden (Etablierung korrekter Rechnungen innerhalb der Spitäler, Kontrollen der Kostenträger, Korrekturen aller fehlerhaften Rechnungen etc.), werden die Kosten-Nutzen-Analysen der Programme entweder ernsthaft gefährden oder werden gar nie in einer Kosten-Nutzen-Analyse auftauchen, da nicht bezifferbar.

\section{Vorschlag der SGPath}

Die SGPath hat einen einfachen und transparenten Vorschlag bzgl. einer zukünftigen Preisgestaltung gemacht. Unserer Erfahrung nach stösst dieser Vorschlag auf grosse Skepsis, da die Kostenträger anscheinend nicht in der Lage sind, die effektiv zu erwartenden Kosten zu antizipieren, und aus verschiedenen, teilweise unverständlichen Gründen nicht bereit sind, neue, und für die Versicherten günstigere, Wege zu beschreiten. Hier hätten wir mehr Bereitschaft für eine Weiterentwicklung des Tarifs erwartet.

Bildnachweis

(C) Panuwat Dangsungnoen | Dreamstime.com

\section{L'essentiel en bref}

- Les cancers colorectaux sont les troisièmes carcinomes les plus fréquents en Suisse en termes d'incidence et de mortalité chez les deux sexes.

- L'objectif prioritaire commun de tous les participants au programme de dépistage est de réduire de manière drastique le nombre élevé de cas de cancer du côlon et de décès, dans une large mesure évitables, en Suisse.

- Un deuxième objectif secondaire devrait être de définir un standard national moderne en matière de processus et d'indemnisation, afin de positionner la Suisse sur le plan international.

- En raison de la structure extrêmement décentralisée en Suisse, tous les acteurs devraient collaborer pour atteindre ces objectifs majeurs. Dans le cas contraire, la population suisse tout entière en pâtirait concrètement. 